\title{
Abstracts for the British Congenital Cardiac Association Annual Meeting: The Barbican, London, 24-25 November 2005
}

\section{Oral Presentations}

Twenty year trends in recognition of lifethreatening neonatal cardiac malformations

Zdenka Reinhardt, Christopher Wren

Department of Paediatric Cardiology, Freeman Hospital, Newcastle upon Tyne

Background: Babies with cardiovascular malformations are usually asymptomatic at birth. Earlier diagnosis is likely to lead to a better outcome. This study examines trends in the timing of diagnosis of potentially life-threatening cardiovascular malformations. Methods: Ascertainment of all cardiovascular malformations diagnosed in infancy in the resident population of one English health region in 1985-2004. Babies considered to have a life-threatening cardiovascular malformation included all those with hypoplastic left heart, pulmonary atresia with intact ventricular septum, transposition of the great arteries, or interruption of the aorta; and babies dying or undergoing operation in the first 28 days with coarctation of the aorta, aortic stenosis, pulmonary stenosis, tetralogy of Fallot, pulmonary atresia with ventricular septal defect, or total anomalous pulmonary venous connection. Results: Of 688,167 live born babies in the 20 years of the study, 4444 had cardiovascular malformations diagnosed in infancy (6.5 per 1000). Cardiovascular malformations were potentially lifethreatening in 685 (15\%). Over the time of the study, $8 \%$ were recognised prenatally, $58 \%$ postnatally before discharge from hospital, $31 \%$ in life after discharge and $3 \%$ after death. Malformations most likely to remain undiagnosed at discharge were coarctation of the aorta $(63 \%)$, interruption of the aortic arch (50\%), and total anomalous pulmonary venous connection ( $47 \%$ ). Over the 20 years of the study, the proportion of babies diagnosed antenatally increased from around $1 \%$ to $20 \%$ and no case was first diagnosed after death in the last 10 years. However, the recent proportion going home without a diagnosis remains around
25\%. Conclusions: Overall one third of babies with potentially life-threatening cardiovascular malformations leave hospital undiagnosed. In recent years better antenatal diagnosis has reduced this proportion to one quarter. Better early recognition of such babies is unlikely to be achieved by clinical examination and is more likely to come from further improvements in antenatal diagnosis and more widespread adoption of routine pulse oximetry.

Early and midterm outcomes of Implantable Cardioverter Defibrillator (ICD) implantation in patients with repaired Tetralogy of Fallot and pulmonary stenosis - a retrospective review

S. Viswanathan, K. English, M. E. C. Blackburn

Department of Paediatric Cardiology, Yorkshire Heart Centre

Introduction: Repair of Tetralogy of Fallot up until recent decades involved aggressive resection and annular enlargement through a right ventriculotomy. This resulted in ventricular scarring and pulmonary incompetence, with an increased risk of ventricular tachyarrhythmia and sudden death in young adulthood. Following the NICE guidelines, implantation of ICDs as primary prevention in patients with repaired Tetralogy is ever increasing. This study aims to determine the rate of appropriate and inappropriate discharges, the success rate of ICD therapy and the impact of ICD implantation on the use of anti-arrhythmic medication in this population of patients. Materials and Methods: This is a retrospective review of patients with repaired Tetralogy of Fallot $(n=18)$ and pulmonary stenosis $(n=2)$ with implantable cardioverter defibrillators managed at our tertiary centre. Patients were identified from our outpatient database, their notes and charts were examined and details regarding indication for ICD implantation, device specifications and complications following implantation were collected. Data 
was also collected on the incidence of appropriate and inappropriate therapies and the success rate of ICD therapy along with the impact of implantation on the usage of anti-arrhythmic medication in these patients. Results: Of the 20 patients, 18 had previous repair of Tetralogy of Fallot and 2 had pulmonary valvotomy and infundibular resection for pulmonary stenosis between 1969 and 1989. 70\% $(n=14)$ of these patients required reoperation with 10 patients having pulmonary valve replacements (PVR), 3 having redo infundibular resections and 1 requiring aortic valve replacement. At the time of consideration for ICD implantation $80 \%$ had moderate to severe pulmonary incompetence and $60 \%$ had more than mild right ventricular dilatation on echocardiography. Indications for ICD implantation were symptomatic ventricular tachycardia requiring cardioversion $(n=8)$, ventricular tachycardia on $24 \mathrm{hr}$ tape/Reveal or electrophysiological study $(n=8)$, ventricular fibrillation (VF)/pulseless ventricular tachycardia (VT) $(n=2)$ and syncope with an abnormal EPS other than VT $(n=2$, high grade ventricular ectopics, sinus node dysfunction).

The median age at implantation was 22 years (16.4-43 years). All our patients had dual chamber devices implanted with either dual $(n=13)$ or single coil $(n=6)$ ventricular leads. GEM3 AT $(n=5)$, Marquis DR $(n=8)$ and Maximo DR $(n=7)$ generators (Medtronic Inc.) were implanted in sub pectoral position and both anti-tachycardia pacing and cardioversion modes were programmed as part of individualised VT and VF protocols. Early post procedural complications included atrial lead displacement $(n=1)$ and pneumothorax requiring drainage $(n=1)$.

During a median follow up of 1.6 years $(0.03-4.5$ years) several episodes of inappropriate therapies were noted in 6 patients $(30 \%)$ especially early after implantation. This was found to be mainly due to atrial tachyarrhythmia, double counting of $\mathrm{T}$ waves or inaccurate interpretation of varying PR intervals as AV dyssynchrony which were effectively dealt with by changes in device programming. There were 33 episodes of inappropriate anti-tachycardia pacing (ATP) in 4 patients and 19 episodes of inappropriate cardioversion in 5 patients. Appropriate ATP was instituted in 4 patients (25\%) with successful termination of all 20 episodes ( $100 \%$ success rate) of ventricular tachycardia. One patient required cardioversion with successful termination of VF. One patient $(5 \%)$ with troublesome tachyarrhythmia died suddenly of unknown cause, 10 months after AICD implantation having had no detections or therapies on his device.

Prior to ICD implantation 8 patients were on amiodarone therapy. At the time of last follow up after AICD implantation all patients were established on anti-arrhythmic agents and of these 6 patients were on amiodarone with the others being effectively managed on beta-blockers and/or flecainide.

Late complications of ICD implantation included lead failure in 1 patient requiring replacement 3.3 years after implantation and generator replacement in a patient who was pacemaker dependent a year after implantation due to an advisory issued by the manufacturer regarding the risk of sudden battery depletion. Conclusions: In our study we found a rate of 0.6 appropriate and 1.4 inappropriate therapies (0.9 episodes of inappropriate ATP and 0.5 episodes of inappropriate cardioversion) per patient-year of follow up following ICD implantation which is in keeping with published literature. The mortality in our study group was 5\% which is acceptable given the high risk population. Implantation of an ICD allowed switching over from amiodarone to less toxic anti arrhythmic therapy in a proportion of patients. Anti-tachycardia pacing was very successful in terminating tachyarrhythmia in our population with $100 \%$ success in terminating ventricular tachycardia.

\section{Treatment with bosentan in children with an intracardiac communication and severe pulmonary arterial hypertension}

P. Bonou, A. A. Hislop, Y. Flynn, S. G. Haworth

\section{Great Ormond Street Hospital and The Institute of Child Health, London, United Kingdom}

Aim: To present the experience of the UK Pulmonary Hypertensive Service for Children at Great Ormond Street Hospital in treating children with "Eisenmenger" type physiology with the dual endothelin receptor antagonist bosentan. Patients and methods: Fourteen inoperable patients were included in the study. These were selected based on a history of an unrestrictive intracardiac communication and established right to left shunt at rest. There was a female : male ratio $1: 0.75$ and age range at initiation of treatment of 7-18.6 years (mean $12.5 \mathrm{yrs}$ ). Six patients had Trisomy 21 . The cardiac diagnoses were: 6 large VSDs, 6 CAVSD (1 of which was not Trisomy 21 ) and 2 more complex congenital heart disease cases that underwent palliative surgery (DILV, DOLV, TGA, PA banding and DORV, TGA, VSD, PS, Waterston). Twelve patients received antiplatelet/ anticoagulation therapy. WHO functional class, haemoglobin $(\mathrm{Hb})$, oxygen saturation, $6 \mathrm{~min}$ walk tests, echocardiograms and ECGs were assessed before starting treatment with bosentan and after 6 and 
12 months of follow up. Twelve patients had a cardiac catheterisation before treatment commenced. The parameters were compared for patients before treatment and at 6 and 12 months post treatment with paired t-test and ANOVA. Results: All patients were severely symptomatic the mean WHO functional class being 3.2 (range 3-4). The mean $\mathrm{Hb}$ was $18.3 \mathrm{~g} / \mathrm{dl}$ (range 13.4-22.9). The mean PVR of all patients was 30.98 units $\mathrm{m}^{2}$ (7.6-57). The Trisomy 21 patients had a mean PVR of 34.4 units $\mathrm{m}^{2}$ and non Trisomy 21 patients had a mean of 28.6 units $\mathrm{m}^{2}$. Mean oxygen saturation at rest was $78.9 \%$ (range 67-93), mean 6 min walk test distance was $223.8 \mathrm{~m}$ $(n=10$, range 108-360) and the lowest oxygen saturation during the walk test was $59.7 \%$ (42-79) at $6 \mathrm{~min}$ or below. Mean duration of therapy was 15.2 months (range 6-34 months). Bosentan was well tolerated in all patients but discontinued in one due to non compliance with monthly screening blood tests. All patients remained stable and their echocardiogram and ECG findings were unchanged throughout. After 6 months mean WHO functional class had decreased significantly to 2.96 ( $\mathrm{p}=0.029)$, whilst $\mathrm{Hb}$ and oxygen saturation at rest had not changed. Ten of the 14 patients were on treatment for more than a year and after 12 months the WHO functional class was maintained at the same improved level. The $\mathrm{Hb}$ and oxygen saturation at rest remained unchanged. After 12 months the 6 min walk mean distance had increased from 211 to 253 metres (non significant). Conclusion: Patients treated with bosentan for up to 1 year showed clinical improvement and remained stable. After 6 months treatment WHO functional class had significantly improved and improvement was sustained for up to 1 year.

\section{Perioperative red cell salvage in paediatric cardiac surgery}

\author{
S. Salam, D. Abrams, A. Kelleher, J. La Rovere
}

Departments of Paediatric Intensive Care and Anaesthesia, Royal Brompton and Harefield NHS Trust, London, United Kingdom

Objective: In recent years blood transfusion has become a debated health care issue. To minimise exposure to infectious agents and reduce bank blood transfusion requirements, leucocyte filtration and perioperative red cell salvage (RCS) are increasingly used in paediatric patients. We hypothesised RCS would reduce the need for additional blood products in children following cardiopulmonary bypass (CPB). Methods: Patients undergoing routine or emergency cardiac surgery requiring $\mathrm{CPB}$ over a study period of 3 months were included prospectively in the analysis.
Haemoglobin, platelet count, coagulation screen and heparin levels were performed before, immediately after surgery and 24 hours later. RCS was performed in theatre according to surgical and anaesthetic preference. Red cells were salvaged from the surgical site, anticoagulated, washed and following resuspension in saline reinfused into the patient within 4 hours. The incidence of post-operative bleeding $(>10 \mathrm{ml} / \mathrm{kg} / \mathrm{hr})$ was recorded, as was the need for additional red blood cells, platelets and fresh frozen plasma (FFP). The need for blood products was at the discretion of the consultant intensivist. Statistical analysis was performed using student t-test and Chi squared methods. Significance was accepted as $\mathrm{p}<0.05$. Results: Thirty-five consecutive patients $(34.54 \pm 43.55$ months, $13.48 \pm 14.39 \mathrm{~kg})$ were included in the analysis. A total of 17 infants $<12$ months were included, 9/24 who received RCS and 8/11 who did not (p 0.052). Cyanotic heart disease was seen in $40 \%$. RCS was performed in 24 of 35 patients, who were significantly older $(44.2 \pm 44.1$ vs. $13.6 \pm 25.5, \mathrm{p} 0.02)$ and heavier $(16.6 \pm 16.2$ vs. $6.7 \pm 4.7, \mathrm{p}$ 0.01). No difference was seen in the prevalence of cyanosis between the two groups. Postoperative bleeding was seen in $21 \%$ who underwent RCS and 40\% in those who did not ( $\mathrm{p} 0.33$ ). The need for additional red blood cells was significantly reduced in those who received RCS, $37.5 \%$ vs. $91 \%$, p 0.003 , as was the use of FFP, $8.3 \%$ vs. $45.5 \%$, p 0.02 . There was no difference in the need for platelet transfusion, $\mathrm{p}$ 0.2. Discussion: In this study RCS was performed on $68.5 \%$ of children following CPB. RCS significantly reduced the need for further blood and FFP transfusion, although this was not related to post-operative bleeding. This has important implications for both exposure to infectious agents and health economics. That children who underwent RCS were older and heavier may be related to the complexity of surgery and $\mathrm{CPB}$ in younger patients, although infants were represented in both groups. A further analysis of potential health and economic benefits in a homogenous group is needed.

\section{Winner of the Young Investigator's Prize}

\section{B-Type Natiuretic Peptide (BNP) as a marker of paediatric heart failure}

\author{
J. Mangat, C. Carter, Y. Foo, M. Burch \\ Cardiothoracic Transplant and Biochemistry Department, \\ Great Ormond Street Hospital, London
}

Background: Paediatric heart failure can be difficult to assess. Symptoms vary widely in children who have 


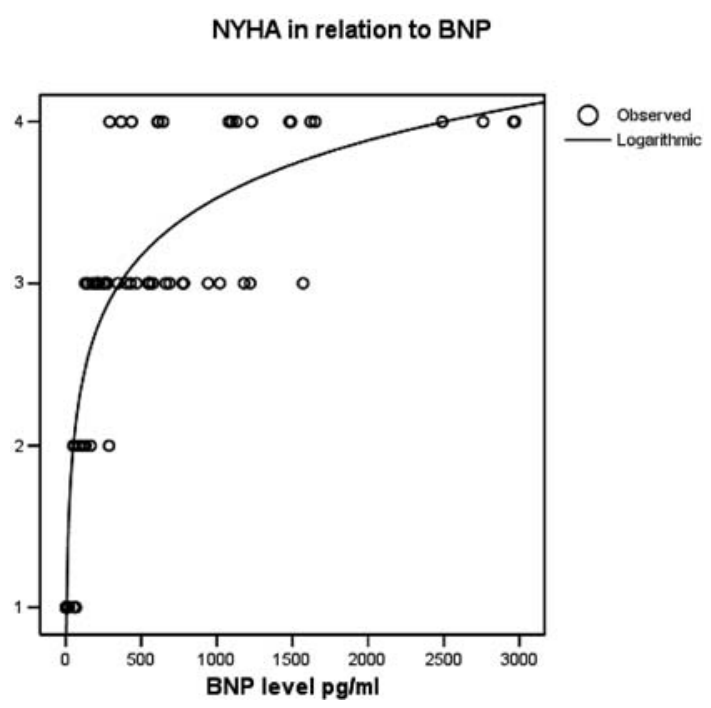

Fraction Shortening (\%) in relation to BNP

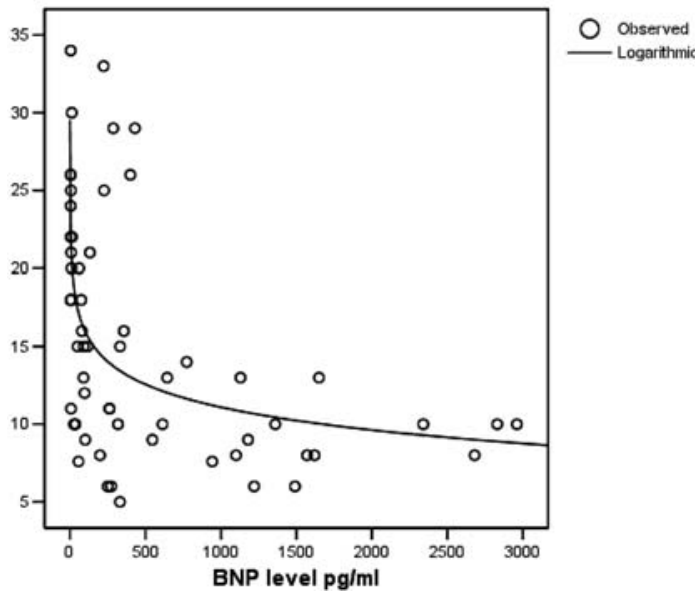

poor ventricular function on echocardiogram. In addition exercise testing is not possible in young children. BNP has been used in adult heart failure and more recently in paediatrics. We investigated BNP in the setting of a paediatric heart failure and transplantation service to assess its usefulness as a clinical marker of heart failure. Method: Clinical and echocardiographic data were correlated to 126 BNP samples. Patients were 3 weeks to 16 years of age. Left ventricular enddiastolic dimension (LVEDd) was related to normal values for body surface area (z-score). Clinical status was defined using Ross and NYHA scores. Thirty four samples were from patients with normal ventricular function. Of the remainder, most were from patients with idiopathic dilated cardiomyopathy (38), anthracycline cardiotoxicity (15), congenital heart disease (25), viral myocarditis (6) and restrictive cardiomyopathy (6). Analysis: BNP was correlated to parametric data (fractional shortening (FS\%)) with pearsons correlation coefficient. For non-parametric data (z-score, NYHA and Ross score), spearmans correlation coefficient was used. Results: Rising BNP levels correlate to deteriorating clinical status with significance to the 0.01 level (Ross and NYHA), Figure 1. There was also correlation, significant to the 0.01 level to FS\%, Figure 2. Increasing BNP levels correlated to the increasing LVEDd z-score significant to the 0.05 level. Mean BNP: in ventricular dysfunction $634 \mathrm{pg} / \mathrm{ml}$ $($ S.E.M. $=80)$, in normal function $11.9 \mathrm{pg} / \mathrm{ml}$ $($ S.E.M. $=1.6)$. Conclusions: This is the largest study of BNP in paediatric heart failure. The stronger correlation of BNP to NYHA and ROSS than to LVEDD and FS\% suggests a useful role in assessment of children with heart failure. We believe it is useful in the outpatient setting particularly when care is shared with general paediatricians. It also appears to be a useful addition in the assessment of heart failure and perhaps timing of transplantation in a specialist centre.

\section{Omega-3 fatty acids - pharmacological preconditioning for paediatric cardiac surgical patients}
J. McGuinness, J. Byrne, A. Hanly, H. Chen, C. Condron, D. Bouchier-Hayes, J. M. Redmond
Department of Surgery, The Royal College of Surgeons in Ireland

Background: Inappropriate systemic inflammatory endothelial and leucocyte activation is a major pathogenic component of post-operative low cardiac output syndrome (LCOS) and associated pulmonary oedema, low urine output, and generalised oedema, in paediatric cardiac surgery. We hypothesised that acute pre-treatment with a parenteral nutrition component omega- 3 fatty acid infusion in-vitro, would prevent the pattern of leucocyte and endothelial activation seen in paediatric cardiac surgery through induction of preconditioning. Methods: Endothelial cells were isolated from saphenous vein of 75 adult cardiac surgery patients, grown in monolayers, pretreated, and then stimulated with either endotoxin (LPS), $\mathrm{TNF}_{\text {alpha }}$, or complement C5a (prime mediators of systemic inflammation with paediatric cardiac surgery). Endothelial production of the neutrophil recruiting cytokines IL-6, IL-8, and endothelial expression of the neutrophil adherence receptors E-Selectin, ICAM-1 were assessed. The effects on the inflammatory pro-coagulant response of the endothelium i.e. loss of surface thrombomodulin and production of tissue factor were also examined. Similar methods were employed using isolated peripheral blood neutrophils. The effects on endothelial nuclear translocation of the acute inflammatory transcription factor $\mathrm{NFkB}$ and the heat shock protein (HSP72) response were investigated for mechanistic insight. Results: Four hours pre-treatment with the omega- 3 infusion 


\begin{tabular}{lccrr}
\hline & Baseline & LPS stimulated & Pre-treated LPS stimulated & p-value \\
\hline IL-6 & $50 \pm 5$ & $2727 \pm 23$ & $211 \pm 8$ & $<0.01$ \\
IL-8 & $26 \pm 16$ & $3678 \pm 10$ & $2650 \pm 36$ & $<0.01$ \\
E-Selectin & $17 \pm 0.2$ & $109 \pm 2$ & $13 \pm 0.4$ & $<0.01$ \\
ICAM-1 & $86 \pm 12$ & $196 \pm 1$ & $72 \pm 0.4$ & $<0.01$ \\
Thrombomodulin & $73.6 \pm 0.7$ & $66.1 \pm 0.3$ & $81.2 \pm 4.9$ & $<0.01$ \\
\hline
\end{tabular}

Units for IL-6, IL-8 are pmol/L

Units for E-Selectin, ICAM-1, and thrombomodulin are mean channel fluorescence on flow cytometry

prevented endothelial release of neutrophil recruiting cytokines IL-6 and IL-8, also prevented upregulation of endothelial receptors required for neutrophil adherence E-Selectin and ICAM-1, and maintained surface anticoagulant thrombomodulin levels, in response to stimulation with LPS (Table 1) or TNF alpha.

The effect of the infusion on adhesion molecule expression was reversible, lasting 18-24 hours. Inflammatory prolongation of neutrophil survival was partially reversed through an increase in apoptotic rate $(73 \% \pm 1 \%$ of control neutrophils apoptotic at 20 hours versus $77 \% \pm 1 \%$ of pre-treated neutrophils, $\mathrm{p}=0.03)$. Pre-treatment produced a more than 2 -fold increase in endothelial HSP72 levels $(p<0.05)$, and reduced nuclear levels of the p65/p50 subunits of $\mathrm{NFkB}$ in response to LPS stimulation $\left(2.1 \pm 0.2 \times 10^{5}\right.$ relative luminescence units in controls versus $1.3 \pm 0.2 \times 10^{5}$ in pre-treated endothelium, $\mathrm{p}<0.01)$. Conclusions: Acute pre-treatment with an omega-3 fatty acid infusion inhibits multiple components of systemic inflammatory activation seen in cardiac surgery. Induction of HSP72 and subsequent reduction of nuclear levels of $\mathrm{NFkB}$, suggest preconditioning mechanisms of inflammatory transcription factor inhibition. This pharmacological strategy may ameliorate paediatric cardiac surgery associated low cardiac output syndrome and it's sequelae.

\section{Poster Presentations}

\section{ECMO support for lifethreatening arrhythmia in infancy permits successful radiofrequency treatment}

A. Rasheed, M. D. Khan, A. K. Duke, M. Tofeig, A. Ng, P. Stafford, F. A. Bu'Lock, R. K. Firmin, G. J. Peek

\section{Department of Paediatric Cardiology, Glenfield Hospital, Groby Road, Leicester}

The majority of arrhythmias presenting in infancy cardiovert readily or rapidly respond to conventional medical therapy. A small number prove highly refractory to anti-arrhythmic medications. Myocardial performance may be severely compromised by the combination of fast heart rate and negatively inotropic drugs. Some babies die. We have recently supported 2 babies with refractory arrhythmias on ECMO, both to pursue drug therapy and eventually, to support the circulation during radiofrequency ablation, with very successful results. The first patient was a $2.5 \mathrm{~kg}$ neonate presenting with collapse secondary to atrial ectopic tachycardia with a rate of 300/minute. Myocardial function was severely impaired. The arrhythmia was adenosine resistant and after iv amiodarone loading had no effect, esmolol infusion was started. This produced profound hypotension and the arrhythmia rapidly recommenced after DC cardioversion. In the face of such severe haemodynamic disturbance, VA ECMO was instituted. Further anti-arrhythmics were tried on-circulatory support, but the arrhythmia was incessant despite multiple DC cardioversions. Therefore radiofrequency ablation of the atrial ectopic focus was attempted on ECMO support. This was achieved uneventfully and the myocardial function rapidly improved, with decannulation 24 hours later. Unfortunately the arrhythmia recurred 2 weeks later, but was successfully treated by further ablation without ECMO. The child remains well with normal development on no medication. An 11-month-old baby presented to the GP with acute onset of lethargy and poor feeding and a heart rate of $350 \mathrm{bpm}$ was noted! This was a broad complex tachycardia with independent $\mathrm{p}$ wave activity (confirmed with adenosine); ie ventricular tachycardia. Although initially well tolerated, the tachycardia resisted DC "cardioversion" even up to $60 \mathrm{~J}$. Progressive and severe myocardial dysfunction and hypotension ensued, exacerbated by any attempts at drug therapy. The patient was therefore placed on VA ECMO. Some slowing of the ventricular rhythm was achieved with amiodarone and flecainide. The patient was then decannulated but the rapid arrhythmia recurred and ECMO was reinstituted. Electrophysiological mapping was then undertaken on ECMO support. NAVEX mapping identified a right ventricular outflow tract focus. This was resistant to conventional RF energy but was eventually successfully ablated with a "Cool-tip" catheter. Myocardial function improved rapidly, the patient was decannulated 
after 48 hours observation and there has been no recurrence of the arrhythmia since discharge. Although viewed as a very invasive technique, VA ECMO support here has prevented two otherwise unavoidable deaths in babies with conditions readily treated by radio-frequency techniques in older children. Not only did ECMO permit institution of aggressive drug therapy but also safely supported catheter interventions in very small patients. ECMO support should be considered early for small patients with refractory arrhythmias, before irreversible neurological compromise ensues. It could also be used electively to permit radiofrequency ablation in children whose size causes concern for safe catheter manipulation.

\section{Epoprostenol treatment in children with pulmonary arterial hypertension}

\author{
A. E. Lammers, ${ }^{1}$ A. A. Hislop, ${ }^{2}$ Y. Flynn, ${ }^{1}$ \\ S. G. Haworth ${ }^{1,2}$ \\ ${ }^{1}$ Great Ormond Street Hospital for Children and \\ ${ }^{2}$ The Institute of Child Health, London, United Kingdom
}

Background: Continuous intravenous Epoprostenol treatment has been shown to be beneficial in patients with severe pulmonary arterial hypertension (PAH). We report the experience of the UK Pulmonary Hypertension Service for Children treating 39 children with this therapy. Patients and methods: The patients were aged 4 months to 17 years (median 5.4 years), male : female ratio was $1: 1.2$. Eleven children were treated with Epoprostenol alone. Twenty-eight were also treated with Sildenafil, Bosentan, Nifedipine or a combination of these. Twenty-five children had idiopathic PAH and 14 had PAH associated with congenital heart disease (9), connective tissue disease (2), chronic lung disease (2) or HIV (1). Cardiac catheter studies made before treatment began showed that in 26 patients the pulmonary artery pressure equalled or exceeded the systemic pressure. Thirty-four patients had a pulmonary vascular resistance (PVR) study with a mean PVR of $23 \pm 12$ units $\mathrm{m}^{2}$. After initial evaluation children were monitored by ECG, transthoracic echocardiography, weight, 6-minute-walk test and WHO functional class. Results: Follow-up was 1-90 months (mean 27). Twenty-four children remain stable and well, 8 have been transplanted and 7 died on treatment. There was no deterioration in the ECG; the 6-minute walk distance and right ventricular function assessed by echocardiography remained stable. WHO functional class improved from 3.6 to 2.6 during the first year $(n=38)(\mathrm{p}<0.001)$ and remained stable for up to 3 years $(n=17)$. Weight improved significantly $(\mathrm{p}<0.05)$. A Kaplan Meier survival curve showed that survival was $94.4 \%$ at 1 year, $90.3 \%$ at 2 years and $83.9 \%$ at 3 years. Conclusions: Epoprostenol treatment improved survival, clinical status and WHO functional class in children with severe PAH. Survival in the children on intravenous Epoprostenol with and without combination therapy was better than that described in adult studies (McLaughlin VV, et al. Circulation 2002; 106: 1477-1482).

\section{Heart transplantation for failure of the systemic right ventricle following atrial inversion procedure (Senning/Mustard) for transposition}
A. A. Lotto, ${ }^{1}$ M. Chaudari, ${ }^{2}$ J. O'Sullivan, ${ }^{2}$ L. Hamilton, ${ }^{1}$ A. Hasan, ${ }^{1}$ J. Dark ${ }^{3}$
${ }^{1}$ Regional Cardiothoracic Centre, ${ }^{2}$ Department of Paediatric Cardiology, ${ }^{3}$ Cardiopulmonary Transplant Unit, Freeman Hospital, Newcastle upon Tyne, United Kingdom

Background: Right ventricular (RV) impairment is a well recognized late sequelae of atrial inversion operation. Although conservative interventions as well as anatomic correction have been advocated late after Mustard or Senning procedures, heart transplantation remains the main treatment of patients with endstage failure of the systemic RV. Aim of this study is to evaluate operative and postoperative results in patients undergoing heart transplantation for endstage failure of the systemic RV following Mustard/ Senning operations. Methods: Retrospective review of patients referred to the Regional Cardiothoracic Centre for cardiac transplantation was performed. Results: Since January 1987, 62 out of 567 patients underwent heart transplantation for congenital heart disease. Nine of these had a previous atrial inversion operation performed (6 Mustard, 3 Senning) for TGA. Mean age at time of atrial inversion operation was $16.9 \pm 10.1$ months. Time interval between atrial inversion and heart transplant was $22.0 \pm 11.6$ years. Mean age at heart transplant was $23.6 \pm 10.8$ years. Three patients were on inotropic support before the operation, while one was on ECMO support. Indication for heart transplantation was end-stage RV faiure (mean NYHA class $3.6 \pm 0.5$ ) with severe impaired function and severe tricuspid valve regurgitation in all patients. Results are shown in Table 1. At a mean follow up time of $21.5 \pm 10.09$ months, $71.5 \%$ of hospital survivors are alive. Conclusions: Heart transplantation for end-stage RV failure following atrial inversion operation is a high-risk procedure; however it remains the main treatment for this group of 
patients. The presence of a failing RV and previous surgery increases the operative mortality and exposes patients to late complications.

\begin{tabular}{ll} 
Mean CPB time & $260.4 \pm 95.3 \mathrm{~min}$ \\
Mean ischaemic time & $214.1 \pm 25.4 \mathrm{~min}$ \\
Mean ventilation time & $79.7 \pm 64.7 \mathrm{hrs}$ \\
Mean ITU stay & $6.5 \pm 4.8$ days \\
Mean postop. stay & $20.5 \pm 8.9$ day \\
In-hospital death & $2 / 9(22.2 \%)$ \\
Late death & $2 / 7(28.5 \%)$ \\
\hline
\end{tabular}

Assessment of the need for elective balloon atrial septostomy in patients with tricuspid atresia. a 25 year experience at the Yorkshire Heart Centre

\author{
D. M. Taliotis J. D. R. Thomson J. L. Gibbs \\ Department of Paediatric Cardiology, \\ Yorkshire Heart Centre
}

Introduction: Significant variation exists in the perceived indications for balloon atrial septostomy in children with tricuspid atresia (TA). Many units advocate early, elective balloon atrial septostomy due to potential progressive narrowing of the interatrial opening, especially after the insertion of an arterial shunt. Our practice is to perform an atrial septostomy only in children with clinical and/or echocardiographic features of a restrictive interatrial communication. The aim of the study was to assess our practice by retrospective evaluation of patients born with tricuspid atresia with particular reference to the clinical fate of the atrial septum. More specifically the frequency of emergency atrial septostomy, the complication rate, the need for delayed septostomy or surgical resection and the longer term outcome were evaluated. Materials and methods: The study was a retrospective review (1980-2005) of the data on 49 live-born patients in our departmental database with a diagnosis of TA. One patient was excluded from the study due to referral to another centre and subsequent loss to follow up. By reviewing the medical notes of the remaining 48 patients we collected data on indications for, and timing of balloon atrial septostomy and subsequent complications and long term outcome. Results: The median time to follow up was 9.5 years $(0.7-23.7)$. The diagnosis group was heterogeneous with $38(79 \%)$ patients diagnosed with TA and normally related great arteries with or without pulmonary stenosis or atresia. The remaining 10 patients had more complex anatomy. Of the 48 patients, $5(10 \%)$ were deemed to require balloon atrial septostomy for a clinically and/or echocardiographically restrictive atrial septum. Three procedures were performed within the first 24 hrs of life and 2 within the first month. One of the patients developed NEC following the septostomy and subsequently died of overwhelming sepsis. One patient required delayed surgical septectomy at 6 years of age, during a bidirectional Glenn operation. Of the remaining 42 patients there were 7 deaths (16\%) but none were attributed to a restrictive atrial septum (1-at birth, severe acidosis and multi organ failure due to interrupted aortic arch and poor response to IV Prostin; 1-5 days with Group B streptococcal sepsis; 1-post operative sepsis following insertion of a BT shunt; 1-9 years, acute shunt obstruction; 1 -withdrawal of care due to complex anatomy deemed to have a poor prognosis; 2 -sudden death at home at $3 \frac{1}{2} 2$ years and 11 years). 26 patients required palliation with an arterial shunt (19-modified BT shunt, 6-Waterston Shunt, 1-ductal stent). None of the patients developed a clinically and/or echocardiographically restrictive atrial septum post procedure or during long term follow-up. Conclusions: We conclude that elective balloon atrial septostomy at presentation may not be necessary in patients born with tricuspid atresia. Accepting the limitations of retrospective data collection we also conclude that progressive narrowing of the interatrial septum at a later date is an unusual occurrence, even after an arterial shunt operation. We recommend that atrial septostomy should only be performed in patients who show obvious clinical and/or echocardiographic evidence of restrictive interatrial communication.

\section{Feasibility and safety of intravascular ultrasound for measurement of coronary atheroma burden in children after heart transplantation}

\author{
Dr Matthew Fenton, Michael Burch
}

\section{Great Ormond Street Hospital for Children NHS Trust, London, United Kingdom}

Purpose: Although intravascular ultrasound (IVUS) in children has been reported it is not widely used due to concerns over safety. Detailed analyses of measurements outlined by the ACC consensus statement on IVUS have not been reported in children. We report our safety and analysis data to date. Procedures: IVUS of the left anterior descending was performed with mechanised pullback concurrently with surveillance coronary angiography. Procedure and fluoroscopy screening times were compared with a second group of patients who experienced coronary angiography alone. Analysis was performed to measure vessel and lumen area, vessel and lumen diameters 


\begin{tabular}{lll}
\hline & Median & Range \\
\hline $\begin{array}{lll}\text { Age at study (years) } \\
\text { Time since transplant (years) }\end{array}$ & $\begin{array}{l}2.3 \\
\text { Donor age }\end{array}$ & $\begin{array}{l}8.9-18.6 \\
0.2-12.1 \\
6-45\end{array}$ \\
\hline & Mean & SD \\
\hline Age at transplant & 11.6 & 4.4 \\
Total length of IVUS pullback (mm) & 49 & 20 \\
Number of slices analysed per study & 21.5 & 6.4 \\
Mean max IMT $>0.5$ (patients) & $8 / 27(30 \%)$ & \\
Max IMT $>0.5$ (patients) & $14 / 27(52 \%)$ & \\
Atheroma burden $>30 \%$ (patients) & $4 / 27(15 \%)$ & \\
\hline
\end{tabular}

\begin{tabular}{lccc}
\hline & $\begin{array}{l}\text { Coronary Angiography } \\
(\boldsymbol{n}=31)\end{array}$ & $\begin{array}{l}\text { IVUS + coronary angiography } \\
(\boldsymbol{n}=27)\end{array}$ & p-value \\
\hline $\begin{array}{l}\text { Procedure time (minutes) } \\
\text { (mean } \pm \text { SD) }\end{array}$ & $31 \pm 15$ & $63 \pm 32$ & 0.08 \\
$\begin{array}{l}\text { Screening time (minutes) } \\
\text { (mean } \pm \text { SD) }\end{array}$ & $7.6 \pm 6.0$ & $11.8 \pm 6.0$ & $<0.01$ \\
\hline
\end{tabular}

and intimal thickness for serial slices at fixed intervals from a distal identification point. Mean atheroma burden, mean maximum intimal thickness (MIT) and overall maximal intimal thickness were determined for each study. Results: Twenty-seven procedures were analysed. Table 1 shows patient demographics and information relating to IVUS analysis. Details of procedure and fluoroscopy time for both IVUS and coronary angiography groups are presented in Table 2 . No complications were encountered. Routine coronary angiography was normal in all but one patient. Conclusions: We have demonstrated the safety of IVUS for coronary surveillance in children. When compared to coronary angiography alone procedure time and screening time are increased but we feel this is outweighed by the increased sensitivity of IVUS. Fiftytwo percent of children have an IMT $>0.5 \mathrm{~mm}$ despite normal angiography. Such detailed analysis would appear central to future research into paediatric coronary disease.

\section{Transcatheter closure of ventricle-pulmonary artery communications after cavopulmonary shunt or Fontan procedure}

\section{T. Desai, J. G. C. Wright, R. Dhillon, O. Stumper \\ Heart Unit, Birmingham Children's Hospital, NHS Trust, Birmingham, United Kingdom}

Background: Ventricle-pulmonary artery connections are rare in patients after the Fontan procedure.
However, these can cause significant long term problems. Hence the catheter occlusion of the anterograde flow in these patients would be beneficial. Further, in patients with a cavopulmonary shunt anterograde pulmonary blood flow is frequently maintained, but, in some patients, can cause significant volume loading of the heart or can complicate the subsequent Fontan procedure. Objective: To evaluate the use of interventional catheter closure of a ventricle-pulmonary artery communication in the setting of a cavopulmonary shunt or after the Fontan procedure. Patients and methods: This was a retrospective study at a tertiary referral centre. Eight patients (Age: 1.5-18 years, mean 7.8 years) underwent transcatheter closure of a ventricle-pulmonary artery communication. Indications were cardiac failure or persistent pleural effusions after cavopulmonary shunt $(n=2)$ or after Fontan $(n=3)$ and abolishing the volume load of the single ventricle prior to Fontan completion $(n=3)$. Results: Devices used were $17 \mathrm{~mm}$ Rashkind Umbrella device $(n=1)$, Amplatzer PDA device $(n=7)$ and Amplatzer ASD device $(n=1)$. One patient required two devices. There were no procedural complications. All 3 patients with prolonged pleural effusions (1 post CP shunt and 2 post Fontan) showed complete resolution of effusions between 4 and 10 days after catheter closure. Two patients underwent transcatheter occlusion for progressive ventricular dilatation and cardiac failure. The first patient was post Fontan and showed gradual improvement in ventricular function. The second patient after $\mathrm{CP}$ shunt died 48 hours post intervention (non procedure related cardiac death). 
Three patients underwent catheter closure to offload the systemic ventricle prior to the Fontan procedure. The device had to be removed prior to release in 1 patient, due to unsatisfactory position. All 3 patients underwent successful completion of Fontan 6 weeks to 3 months post catheter. Conclusions: Transcatheter closure of ventricle-pulmonary artery communication is a safe and effective technique in the treatment of selected patients after CP shunt or Fontan procedure with early or late complications due to inappropriate pulmonary blood flow. This intervention should also be considered prior to the Fontan procedure in selected patients with ventricular overload.

\section{Severe coronary ostial stenosis after arterial switch operation detected by transthoracic Doppler echocardiography}

\author{
E. H. Aburawi, ${ }^{1}$ A. Berg, ${ }^{2}$ H. Arheden, ${ }^{3}$ \\ M. Karlsson, ${ }^{3}$ P. Jögi, ${ }^{4}$ E. Pesonen ${ }^{1}$ \\ Departments of ${ }^{1}$ Paediatric Cardiology, ${ }^{4}$ Cardiac \\ surgery, and ${ }^{3}$ Clinical Physiology, Lund University \\ Hospital, Lund, Sweden; Department of Paediatrics, \\ ${ }^{2}$ Institute of Clinical Medicine Bergen University \\ Hospital, Norway
}

Background: Asymptomatic proximal coronary artery stenosis after arterial switch operation (ASO) is rare, but a potentially life-threatening condition, that is reported to appear in up to $7 \%$. Angiography, although considered the state-of-the-art method of diagnosis, is an invasive method, but has limitations for diagnosing ostial stenosis. We report changes in Doppler flow profile and coronary flow reserve (CFR) in two asymptomatic patients (9-and 10-year old) with left main coronary artery (LMCA) ostial stenosis after ASO. Methods: Coronary flow was assessed by Transthoracic pulsed and colour-flow Doppler echocardiography (TTDE). CFR was measured in one patient using adenosine infusion $(140 \mathrm{mcg} / \mathrm{kg} / \mathrm{min})$ over 4 minutes. CFR was calculated as the ratio of reactive hyperaemia to basal average peak velocity (APV). Both children were investigated with coronary angiography. They had myocardial Single-photon Emission Computed Tomography (SPECT), and magnetic resonance imaging (MRI) at rest and after reactive hyperaemia with adenosine infusion. Both patients had balloon dilatation and Cypher select (drug eluted) stent. Results: On echocardiogram a flame-like colour-flow diastolic signal was detected at the stenotic coronary ostia. The maximal spectral velocities during baseline conditions over the stenotic ostia were over 1.9 and $2.0 \mathrm{~m} / \mathrm{s}$ (normal $30 \pm 10 \mathrm{~cm} / \mathrm{s}$ ). The post-stenotic
CFR was haemodynmically significant with value of 1.3 , normal adult range $2.5-4$. Coronary angiography showed a significant ostial stenosis $90 \%$ in both patients. Myocardial SPECT and MRI at rest/adenosine infusion were consistent with severe myocardial ischemia in the territory of the left coronary artery. Normal coronary angiography and coronary flow studies after stenting. Conclusion: We suggest that coronary artery flow assessment should be an integral part of the TTDE in the follow up of children with ASO. Serious coronary artery stenosis can be detected with TTDE. Assessment of CFR provides information of the physiological significance of the coronary stenosis.

8 Years of fetal echocardiography in high-risk mothers: The Birmingham Women's Hospital experience

S. V. Rasiah, ${ }^{1}$ A. K. Ewer, ${ }^{1}$ P. Miller, ${ }^{2}$ J. G. Wright ${ }^{2}$, M. D. Kilby ${ }^{3}$

${ }^{1}$ Department of Neonatology and ${ }^{3}$ Department of Fetal Medicine, Birmingham Women's Hospital; ${ }^{2}$ Department of Paediatric Cardiology, Birmingham Children's Hospital

Introduction: Congenital heart disease (CHD) affects 8 per 1000 live births and it is also responsible for $20 \%$ of neonatal deaths. Antenatal diagnosis of major CHD allows appropriate counselling and planning for delivery at a neonatal unit with appropriate intensive care and transport facilities. Birmingham Women's Hospital provides a supra-regional specialist fetal echocardiography in high-risk mothers. Aim: To evaluate fetal echocardiography findings in highrisk mothers over an 8 year period. Method: We undertook a retrospective review of all pregnant women at high-risk of having a baby with congenital heart disease who underwent fetal echocardiography between 01/01/1997 and 31/12/2004 at Birmingham Women's Hospital. Results: 3,963 mothers were referred for fetal echocardiography and a total of 5,568 fetal echocardiography examinations were carried out during this period. The main reasons for referral were: (i) previously affected child $-27 \%$ (ii) abnormal initial screening scan - $20.7 \%$ (iii) maternal cardiac condition $-9.5 \%$ (iv) infant of diabetic mothers $8 \%$ and (v) increased fetal nuchal translucency $-3 \%$. Seven hundred and twleve (17.9\%) echocardiograms were reported as abnormal. The majority of the abnormalities were identified in mothers who had abnormal initial screening scan (62\%). In addition, the echocardiogram was also abnormal in $9 \%$ of cases with increased fetal nuchal translucency and in $5.7 \%$ of 
infants of diabetic mothers. In those with previously affected child and maternal cardiac condition, the echocardiogram was abnormal in $2.5 \%$ and $2.6 \%$ respectively. Conclusion: Abnormal initial screening scans and increased nuchal translucency had the highest yield in identifying CHD in high-risk mothers. Infant of diabetic mothers also have an increased risk warranting fetal cardiac screening for CHD. Normal fetal echocardiogram provides reassurance for the remainder of parents. 